\title{
Are Analogue or Digital Clocks Friendlier for People Living with Dementia?
}

\author{
Akihiro Koreki $^{\mathrm{a}}$ Keisuke Kusudo ${ }^{\mathrm{a}}$ Hisaomi Suzuki ${ }^{\mathrm{a}}$ Shoko Nozaki ${ }^{\mathrm{a}}$ \\ Mitsumoto Onaya $^{a}$ Alison Bowes $^{b}$ Mitsuhiro Sado ${ }^{c}$ \\ aDepartment of Psychiatry, National Hospital Organization Shimofusa Psychiatric Medical Center, Chiba, Japan; \\ ${ }^{b}$ Faculty of Social Sciences, University of Stirling, Stirling, UK; ' Department of Neuropsychiatry, Keio University \\ School of Medicine, Tokyo, Japan
}

\author{
Keywords \\ Clock · Dementia · Delirium · Environment · Design · \\ Prevention
}

\begin{abstract}
Background: In ageing population, it is desirable to reduce the impact of cognitive decline on daily life. While various types of dementia-friendly environments have been proposed, the question still remains regarding whether analogue or digital clocks are friendlier for people with dementia. Methods: In clinical practice, we normally use our original clock reading test (10 analogue and 10 digital clocks) to assess patients' ability to read a clock. In the present study, a retrospective medical record survey was conducted. Fiftyfive participants who had done the test were identified. The result of the test was compared between analogue and digital clocks. Additionally, to assess specific ability to read analogue clocks, an "analogue-digital gap" was defined as the difference between patients' performance for analogue and digital clocks. Univariate and multivariate analyses were conducted to detect significant factors associated with reading ability specific to analogue clocks. Results: The analogue clock proved less readable than the digital clock, even after
\end{abstract}

karger@karger.com www.karger.com/dee

Karger $\stackrel{\text { ' }}{5}$

BOPEN ACCESS
(C) 2021 The Author(s)

Published by S. Karger AG, Basel

This is an Open Access article licensed under the Creative Commons Attribution-NonCommercial-4.0 International License (CC BY-NC) (http://www.karger.com/Services/OpenAccessLicense), applicable to the online version of the article only. Usage and distribution for commercial purposes requires written permission. adjusting for MMSE total score $(p=0.003)$. Multivariate analysis revealed reading ability of the analogue clock was significantly associated with MMSE calculation and clock drawing test ( $p=0.009$ and 0.040 , respectively). Conclusions: In the present study, the digital clock was friendlier than the analogue clock for patients with dementia. Compared to the digital clock, reading analogue clocks might require more widespread cognition, such as working memory and visuospatial processing. While our finding was a general tenden$c y$, and individual assessment is necessary, it might help the development of personalized environmental adjustments.

\footnotetext{
(c) 2021 The Author(s).

Published by S. Karger AG, Basel
}

\section{Introduction}

Population ageing is one of the most critical global social challenges, and as a consequence of ageing, the number of people with dementia across the world is reportedly over 50 million in 2019 and will increase to 152 million by 2050 [1]. Therefore, there is an urgent need to reduce the impact of cognitive decline due to both ageing and dementia on daily life. In current societies, 
environments designed for people with physical decline, such as step-free access and availability of elevators, are already widespread. In contrast, environment designed for people with cognitive decline has not yet been fully implemented. Recently, various types of dementia-friendly designs have been proposed, in areas including bedrooms, bathrooms, toilets, lounges, entrances, gardens, and public places [2]. However, although particular clock designs are often recommended, there is little systematic research on the relative merits of different types of clocks, specifically the choice of analogue or digital clocks. We consider this question crucial because of the importance of clock time orientation and understanding in maintaining normal daily life in modern contexts.

Recognition of current time is one of the most important cognitive skills for human life. If a person lives without clocks, their circadian rhythm can be disrupted, and this can result in various kinds of mental problems [3]. Particularly, circadian rhythm disruptions are common in older people and are more severe in people with dementia $[4,5]$. A clock is one of the most important items for people with delirium to mitigate their symptoms [6, 7]. In addition, circadian rhythm disruptions might also be a potential risk factor for developing dementia $[4,5]$. Although light has a much stronger effect on circadian rhythm, recognition of current time using clocks might also be considered a key factor for maintaining good mental health in people with dementia.

In response to the importance of clocks, our routine clinical assessment includes our original clock reading test and traditional cognitive assessments, such as MMSE and clock drawing/copying tests. Our original clock reading test consists of 10 analogue clocks and 10 digital clocks which are used to assess the ability to read each type of clock (detailed procedure in the Methods section). It helps us to develop a personalized environment for each patient by identifying the most appropriate type of clock for them to use at home and in hospital rooms. In the present study, we analysed these clinical assessments to investigate the question of whether an analogue or a digital clock was more helpful for people with dementia and an advanced question regarding what kind of factors were associated with their ability to read clocks. Particularly, as there is convergent evidence regarding difficulties both with drawing and reading analogue clocks in the Alzheimer's disease [8-10], we had a hypothesis that digital clocks might be friendlier for patients with dementia.

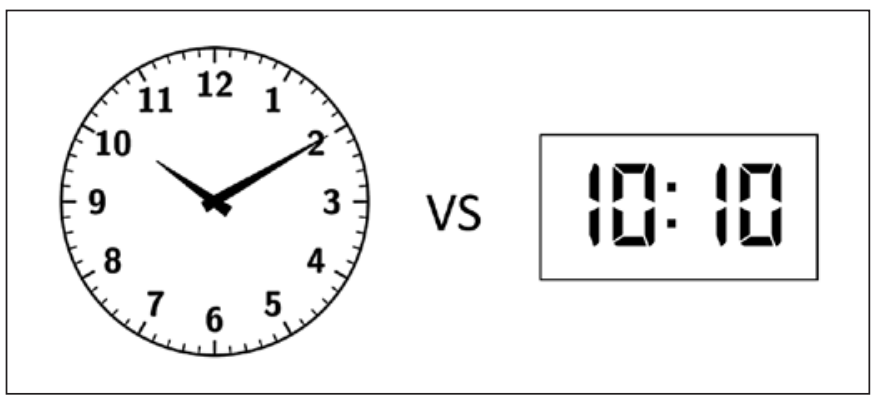

Fig. 1. Our original clock reading task consists of 10 analogue clocks and 10 digital clocks to assess which ability to read has declined less.

\section{Methods}

The present study was conducted as a retrospective medical record survey, and the data were obtained from our medical records during October 2019-September 2020 at Shimofusa Psychiatric Medical Center, Japan. The data included patient characteristics (i.e., age, sex, and education level), Mini-Mental Scale Examination (MMSE) [11], Functional Assessment Staging Tool (FAST) [12], Clinical Dementia Rating (CDR) [13], reverse fox test [14], cube copying test [15], clock drawing/copying test [8], and our original clock reading test. Our clock reading test consists of 10 analogue clocks and 10 digital clocks used to assess which ability to read has declined less (see Fig. 1). First, patients were asked to read each analogue clock and then each digital clock. The order of each type of clock was pseudo-randomized. As the main purpose of the task was to assess ability to read clocks in daily life and to give some advice regarding their environment, the analogue clock face showed all numbers. This is different from a traditional clock reading task, in which numbers are not shown [10]. We used a clock with numbers because it is more appropriate for assessing reading ability in the real world. In addition, we allowed experimenters to support patients' reading to compensate for poor attention. This was done to minimize the effects of mistakes caused by inattention.

In the present retrospective survey, the inclusion criteria were patients who had undergone at least the clock reading test. The exclusion criteria were patients without dementia-related conditions, that is, patients whose cognitive impairment could be explained from their mood disorder or schizophrenia itself and was not considered any potential prodromal symptom of dementia. As our hospital is a psychiatric hospital without any surgical department, no patients had postoperative delirium. Fifty-five participants were included (mean age: $81.6[ \pm 8.6]$, female $=27$, inpatient $=19$, MMSE = 16.4 $[ \pm 5.7])($ Table 1$)$. Their dominant dementia types were diagnosed based on their history of illnesses, clinical follow-up, brain imaging, and neuropsychological tests except the clock reading test (i.e., clock reading test was used only for the assessment of their ability to read clocks to manage their environment, not for diagnosis) and were confirmed by 2 BoardCertified Dementia practitioners of the Japanese Society of Psychiatry and Neurology (A.K. and H.S.) according to the Diagnostic and Statistical Manual of Mental Disorder, fifth edition. In re- 
Table 1. Characteristics of patients $(n=55)$

Age, years, mean $( \pm \mathrm{SD})$

Sex, male/female, $N$

Educational level, years, mean $( \pm S D)$

Outpatient/inpatient, $N$

MMSE, mean $( \pm S D)$

Dementia type, $N$

$\begin{array}{lr}\mathrm{AD} & 21 \\ \mathrm{VaD} & 17 \\ \mathrm{DLB} & 8 \\ \text { FTD } & 1 \\ \text { Others } & 8\end{array}$

MMSE, Mini-Mental Scale Examination; AD, Alzheimer's disease; $\mathrm{VaD}$, vascular dementia; DLB, dementia with Lewy bodies; FTD, frontotemporal dementia. sponse to the current understanding of mixed brain pathology in dementia $[16,17]$, their most dominant dementia type was analysed in the present study. Their dominant dementia types were diagnosed as Alzheimer's disease ( $\mathrm{AD}, n=21)$, vascular dementia (VaD, $n=17$ ), dementia with Lewy bodies (DLB, $n=8$ ), frontotemporal dementia $(n=1)$, Korsakoff syndrome $(n=2)$, dementia not otherwise specified $(n=1)$, and mild cognitive impairment (MCI, $n=5$ ).

To compare ability to read analogue and digital clocks ( $\max 10$ each), we conducted repeated measures analysis of covariance with MMSE total score as a covariate because there are 2 different conditions (analogue and digital) within 1 participant. Next, to investigate the differences in performance among dementia types $(\mathrm{AD}$, $\mathrm{VaD}, \mathrm{DLB}$, and others), a Kruskal-Wallis test and a subsequent post hoc analysis were conducted. Finally, to investigate significant factors related to clock reading ability, especially analogue clocks, we began by calculating the analogue-digital gap, based on scores in each type of clock reading task. For example, if the score for analogue clock reading was 6 and digital was 10 , the gap was 4 . As there were many factors potentially associated with clock reading ability, such as MMSE subscale, we analysed the association between the analogue-digital gap and potential factors using univariate analyses, and subsequently, we conducted a stepwise logistic regression analysis as a multivariate analysis with the analoguedigital gap as a dependent variable and only factors that showed significance in univariate analysis except MMSE total score as independent variables. Univariate analyses were conducted depending on the data type and distribution. In a logistic regression analysis, because the analogue-digital gap and MMSE subscales were not normally distributed, they were converted to binary data using median split. All analyses were conducted with R (4.0.2), which is the most widespread and powerful software environment for statistical computing, and $p<0.05$ was considered significant. In a post hoc analysis of comparison between dementia types, a false discovery rate was used.



Fig. 2. Comparison of reading abilities between analogue and digital clocks revealed that the digital clock was more readable for patients with dementia than the analogue clock after the adjustment of MMSE total score $(p=0.003)$.

\section{Results}

Our analysis revealed that the digital clock was more readable for patients with dementia than the analogue clock after the adjustment of MMSE total score $(p=0.003)$ (Fig. 2), with a finding that more severe cognitive impairment was linked to a greater analogue-digital gap. There was a significant difference in the analogue-digital gap among dementia types $(p=0.018)$, and the post hoc analysis revealed that $\mathrm{AD}$ showed greater gap than others $(p=0.001)$ and DLB also showed greater gap than others $(p=0.009)$ (Fig. 3). In the univariate analyses, the analogue-digital gap was significantly associated with CDR $(p=0.046)$, MMSE total $(p=0.003)$, time orientation $(p=0.040)$, place orientation $(p=0.024)$, immediate re- 


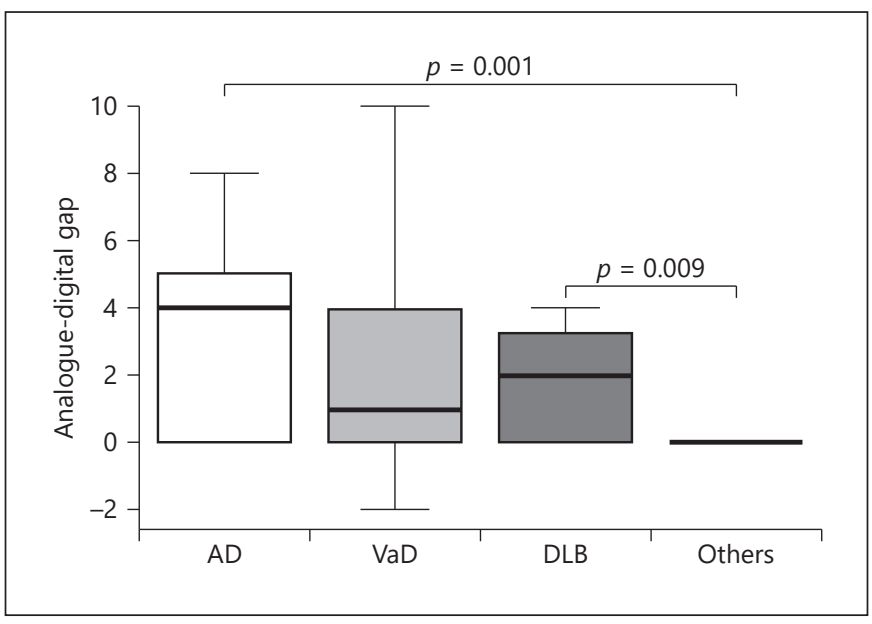

Fig. 3. Patients with $A D$ and DLB showed significantly impaired reading ability of analogue clocks. $\mathrm{AD}$, Alzheimer's disease; $\mathrm{VaD}$, vascular dementia; DLB, dementia with Lewy bodies; FTD, frontotemporal dementia.

call $(p=0.017)$, calculation $(p<0.001)$, naming objects $(p=0.013)$, clock drawing test $(p<0.001)$, clock copying test $(p=0.010)$, and reverse fox test $(p=0.047)$, while other factors did not reach significance (Table 2). In the multivariate analysis, CDR, time orientation, place orientation, immediate recall, calculation, naming objects, clock drawing test, clock copying test, and reverse fox test were initially included as independent variables in our stepwise logistic regression analysis. After the stepwise procedure, our model finally selected calculation and clock drawing test as dependent variables $(\mathrm{AIC}=60.4)$, revealing that the analogue-digital gap ( $\geq 2$ or not) was significantly associated with difficulty in the MMSE calculation ( $\leq 1$ or not) (odds ratio [OR] 9.9 [95\% confident interval 2.1-73.0], $p=0.009$ ) and clock drawing test $(\mathrm{OR}=0.8[0.6-0.98], p=0.040)$ remained significant.

\section{Discussion}

This is the first report addressing the question regarding which of analogue or digital clocks is more readable for people with dementia. Our analysis showed that the digital clock was friendlier for patients with dementia. In addition, impaired reading ability of the analogue clock relative to the digital clock was seen in AD and DLB. Reading ability was significantly associated with abilities to do mental arithmetic and draw a clock.
Table 2. Analogue-digital gap and associated factors

\begin{tabular}{llr}
\hline Univariate analyses & Statistic & $p$ value \\
\hline Age & rho $=0.05$ & 0.708 \\
Sex & $U=326.5$ & 0.374 \\
Educational level & rho $=-0.24$ & 0.088 \\
Outpatient/inpatient & $U=366.5$ & 0.660 \\
$\quad$ FAST & rho $=0.09$ & 0.496 \\
CDR & rho $=0.27$ & $\mathbf{0 . 0 4 6}$ \\
MMSE total & rho $=-0.39$ & $\mathbf{0 . 0 0 3}$ \\
Time orientation & rho $=-0.28$ & $\mathbf{0 . 0 4 0}$ \\
Place orientation & rho $=-0.30$ & $\mathbf{0 . 0 2 4}$ \\
Immediate recall & rho $=-0.32$ & $\mathbf{0 . 0 1 7}$ \\
Calculation & rho $=-0.51$ & $<\mathbf{0 . 0 0 1}$ \\
Delayed recall & rho $=-0.11$ & 0.406 \\
Naming objects & rho $=-0.33$ & $\mathbf{0 . 0 1 3}$ \\
Repeating sentence & $U=435$ & 0.240 \\
Commands & rho $=0.07$ & 0.599 \\
Reading & $U=251$ & 0.305 \\
Writing & $U=357.5$ & 1.000 \\
Intersecting pentagon copy & $U=432.5$ & 0.318 \\
Clock drawing test & rho $=-0.45$ & $<\mathbf{0 . 0 0 1}$ \\
Clock copying test & rho $=-0.35$ & $\mathbf{0 . 0 1 0}$ \\
Reverse fox test & $U=452$ & $\mathbf{0 . 0 4 7}$ \\
Cube copying test & rho $=-0.25$ & 0.078 \\
\hline Multivariate analysis & OR $(95 \%$ CI $)$ & $p$ value \\
\hline Analogue-digital gap $\geq 2)$ & & \\
Difficulty in the calculation $(\leq 1)$ & $\mathbf{9 . 9}[\mathbf{2 . 1 - 7 3 . 0 ]}$ & $\mathbf{0 . 0 0 9}$ \\
Clock drawing test & $\mathbf{0 . 8}[\mathbf{0 . 6}-\mathbf{0 . 9 8}]$ & $\mathbf{0 . 0 4 0}$ \\
\hline & & \\
\hline & &
\end{tabular}

In the multivariate analysis, analogue-digital gap and MMSE subscales were converted to binary data using median split. A stepwise logistic regression analysis was done with significant factors in univariate analyses as dependent variables. FAST, Functional Assessment Staging Tool; CDR, Clinical Dementia Rating; MMSE, Mini-Mental Scale Examination; OR, odds ratio; $\mathrm{CI}$, confidence interval.

Our findings are in line with existing studies regarding the clock reading task. Schmidtke and Olbrich [10] revealed difficulties in reading analogue clocks in patients with AD and DLB as well as patients with parietal lobe lesions. Fukui and Lee [9] also demonstrated difficulties in reading analogue clocks in patients with $\mathrm{AD}$, and their difficulties were associated with their limitations in basic and instrumental activities of daily living. Saur et al. [18] completed a functional magnetic resonance imaging study of the clock reading task in both healthy controls and patients with $\mathrm{MCI} /$ early $\mathrm{AD}$, illustrating lower performance in patients and quadratic correlation between their performance and task-related brain activations in the ventral visual stream and precuneus known to be in- 
volved in conceptual processing and spatial imagery, respectively. As the precuneus is one of the regions where $\mathrm{A} \beta$ deposition and atrophy occur first in $\mathrm{AD}$, it is reasonable that faster decline of ability to read analogue clocks than digital clocks was seen in patients with dementia, especially $\mathrm{AD}[19]$.

Both abilities to do mental arithmetic and draw a clock were significantly associated with specific difficulties in reading analogue clocks. MMSE calculation is characterized by mental arithmetic, requires working memory beyond simple attention [20], and is associated with brain activation in both frontal and parietal regions including the precuneus [21]. Compared to digital clock reading, reading the analogue clock with numbers in the present study, especially the minute hand, may require working memory for transformation from location of both hands and actual number on a clock face to current time. Indeed, a study in children revealed that children with mathematics difficulties performed significantly worse than other children on clock reading tasks [22]. Drawing a clock also requires multiple cognitive functions, such as semantic memory, visuospatial processing, and executive function, and is associated with a wide range of brain activations including frontal and parietal regions [23]. As both clock reading and drawing tests require semantic memory and visuospatial processing of analogue clocks, the link is consequent.

Our starting point in this study was how to reduce the impact of cognitive decline on the patient's life, rather than differential diagnostic use. One of the most distressing symptoms in dementia is delirium. There might be interrelationships between dementia and delirium, that is, dementia is the prominent risk factor for delirium and delirium is an independent risk factor for subsequent dementia because delirium itself can cause permanent neuronal damage [24]. Therefore, prevention or mitigation of delirium is a significant issue. Circadian rhythm disruption is highly related to delirium. It is a core domain of delirium and also the leading contributing factor of delirium [25]. Circadian rhythm disruptions are severe in people with dementia $[4,5]$, and recognition of current time using clocks is helpful for preventing or mitigating delirium $[6,7]$. Our finding suggests that the digital clock might be better for people with dementia who are at risk of delirium. However, personalizing is important, and individual clinical assessment regarding clock reading ability is necessary.

There were several limitations. First, our sample may be highly biased because our psychiatric hospital has a role as a tertiary referral hospital, and a half of patients were referred to our hospital with the purpose of hospi- talization. In addition, patients with severe dementia who could not perform the tests were excluded. Further research is necessary to generalize our findings. Second, this study was a cross-sectional study, and it was impossible to assess the longitudinal effect of maintaining clock reading. Third, as our hospital was a psychiatric hospital, most patients have behavioural and psychological symptoms of dementia (BPSD). Although the assessment was conducted after patients' symptoms improved to the clinical level that they could perform the tests, it cannot be completely denied that their symptoms and drugs could affect their performance. However, there was no significant difference between inpatients and outpatients, and comparison between analogue and digital clocks could control their general effect of BPSD. Furthermore, our study was conducted in a clinical, not an experimental setting, and it may be easier to apply our finding in such clinical settings where patients usually show BPSD and have several medications. Fourth, there still remains a question regarding clocks that have both analogue and digital faces. This kind of a mixed clock might be helpful for patients with dementia or might be confusing for them. Further research is required to answer this question. Fifth, there could be a difference between reading and recognizing. In our assessment, the meaning of the current clock time was not asked, such as time to eat breakfast and time to sleep. Although recognizing current time should work based on reading clocks, there might remain a possibility that patients can recognize meaning of current time without reading it, because information processing in the brain might differ between them.

In conclusion, the digital clock was friendlier than the analogue for our sample, and the ability to read analogue clocks might be associated with both working memory and visuospatial processing. While our finding was a general tendency and individual assessment is important to develop personalized environment, it provided evidence for dementia-friendly design regarding clocks.

\section{Acknowledgments}

We thank all patients and the staff for supporting this study.

\section{Statement of Ethics}

Ethical approval was obtained from the Institutional Ethical Review Board of the Shimofusa Psychiatric Medical Center (020917002). The study was carried out in accordance with the ethical guidelines set forth by the Declaration of Helsinki. 


\section{Conflict of Interest Statement}

The authors report no conflicts of interest regarding this study.

\section{Funding Sources}

The authors report no sources of funding regarding this study.

\section{Author Contributions}

A.K. designed the study and conducted data collection. A.K. and K.K. analysed the data. A.K. and H.S. checked patients' diagnoses. S.N. and M.O. developed research environment. A.B. and M.S. provided technical and conceptual suggestions. A.K. wrote the first draft, and all co-authors checked it.

\section{Data Availability Statement}

The datasets are available from the corresponding author on reasonable request.

\section{References}

1 Alzheimer's Disease International. World alzheimer report 2019. The global impact of dementia analysis of prevalence, incidence, cost \& trends. London: Alzheimer's Disease International; 2019.

2 Bowes A, Dawson A. Designing environments for people with dementia: a systematic literature review. Emerald publishing; 2009. p. 1-94.

3 Ahmad M, Md Din NSB, Tharumalay RD, Che Din N, Ibrahim N, Amit N, et al. The effects of circadian rhythm disruption on mental health and physiological responses among shift workers and general population. Int $J$ Environ Res Public Health. 2020;17:7156.

4 Leng Y, Musiek ES, Hu K, Cappuccio FP, Yaffe K. Association between circadian rhythms and neurodegenerative diseases. Lancet Neurol. 2019;18:307-18.

5 Musiek ES, Holtzman DM. Mechanisms linking circadian clocks, sleep, and neurodegeneration. Science. 2016;354:1004-8.

6 McCusker J, Cole M, Abrahamowicz M, Han L, Podoba JE, Ramman-Haddad L. Environmental risk factors for delirium in hospitalized older people. J Am Geriatr Soc. 2001;49: 1327-34.

7 Preventing delirium in the hospital. Clocks and calendars may help patients stay oriented. Harv Health Lett, 2011;36:4

8 Rouleau I, Salmon DP, Butters N, Kennedy C, McGuire K. Quantitative and qualitative analyses of clock drawings in Alzheimer's and Huntington's disease. Brain Cogn. 1992;18: $70-87$.
9 Fukui T, Lee E. Visuospatial function is a significant contributor to functional status in $\mathrm{pa}$ tients with Alzheimer's disease. Am J Alzheimers Dis Other Demen. 2009;24:313-21.

10 Schmidtke K, Olbrich S. The clock reading test: validation of an instrument for the diagnosis of dementia and disorders of visuo-spatial cognition. Int Psychogeriatr. 2007;19: $307-21$.

11 Folstein MF, Folstein SE, McHugh PR. "Minimental state". A practical method for grading the cognitive state of patients for the clinician. J Psychiatr Res. 1975;12:189-98.

12 Sclan SG, Reisberg B. Functional assessment staging (FAST) in Alzheimer's disease: reliability, validity, and ordinality. Int Psychogeriatr. 1992;4 Suppl 1:55-69.

13 Hughes CP, Berg L, Danziger WL, Coben LA, Martin RL. A new clinical scale for the staging of dementia. Br J Psychiatry. 1982;140:56672.

14 Tabuchi H, Konishi M, Saito N, Kato M, Mimura M. Reverse Fox test for detecting visuospatial dysfunction corresponding to parietal hypoperfusion in mild Alzheimer's disease. Am J Alzheimers Dis Other Demen. 2014;29:177-82.

15 Maeshima S, Osawa A, Maeshima E, Shimamoto Y, Sekiguchi E, Kakishita K, et al. Usefulness of a cube-copying test in outpatients with dementia. Brain Inj. 2004;18:889-98.

16 Coulthard EJ, Love S. A broader view of dementia: multiple co-pathologies are the norm. Brain. 2018;141:1894-7.
17 Alafuzoff I, Libard S. Mixed brain pathology is the most common cause of cognitive impairment in the elderly. J Alzheimers Dis. 2020;78:453-65.

18 Saur R, Milian M, Erb M, Eschweiler GW, Grodd W, Leyhe T. Cortical activation during clock reading as a quadratic function of dementia state. J Alzheimers Dis. 2010;22:26784.

19 Jagust W. Imaging the evolution and pathophysiology of Alzheimer disease. Nat Rev Neurosci. 2018;19:687-700

20 Bristow T, Jih CS, Slabich A, Gunn J. Standardization and adult norms for the sequential subtracting tasks of serial 3's and 7's. Appl Neuropsychol Adult. 2016;23:372-8.

21 Hawes Z, Sokolowski HM, Ononye CB, Ansari D. Neural underpinnings of numerical and spatial cognition: an fMRI meta-analysis of brain regions associated with symbolic number, arithmetic, and mental rotation. Neurosci Biobehav Rev. 2019;103:316-36.

22 Burny E, Valcke M, Desoete A. Clock reading: an underestimated topic in children with mathematics difficulties. J Learn Disabil. 2012;45:351-60.

23 Talwar NA, Churchill NW, Hird MA, Pshonyak I, Tam F, Fischer CE, et al. The neural correlates of the clock-drawing test in healthy aging. Front Hum Neurosci. 2019;13:25.

24 Fong TG, Davis D, Growdon ME, Albuquerque A, Inouye SK. The interface between delirium and dementia in elderly adults. Lancet Neurol. 2015; 14:823-32.

25 Daou M, Telias I, Younes M, Brochard L, Wilcox ME. Abnormal sleep, circadian rhythm disruption, and delirium in the ICU: are they related? Front Neurol. 2020;11:549908. 